\title{
The Use of Thoracic Epidural for Rapid Opioid Taper
}

\author{
Samuel P. Ang, MD¹, Martha L. Castro, MD², Michael Montuori, MD, Feza Remzi, MD³, Shengping Zou, MD',
} and Steven Calvino, MD'

Background: Inflammatory Bowel Disease (IBD) is often associated with significant abdominal pain that can be challenging to control. Although controversial, opioids are often prescribed for the management of abdominal pain in patients with IBD. There have been several methods described for the rapid taper of patients on long-term, high-dose opioids. However, to date, there have been no reported cases using epidural analgesia for rapid opioid taper.

Case Report: We present a case of a 36-year-old man with ulcerative colitis and recurrent bowel obstructions on a high-dose transdermal fentanyl patch whose opioid consumption was rapidly tapered during inpatient hospitalization utilizing thoracic epidural analgesia.

Conclusion: The potential role of epidural analgesia in rapid opioid taper has yet to be explored. In patients with chronic pain and inflammatory bowel disease or recurrent bowel obstructions, epidural analgesia may be particularly helpful to improve gastrointestinal motility while also being used to rapidly taper opioid dosage.

Key words: Inflammatory bowel disease, ulcerative colitis, Crohn's disease, epidural, opioids, rapid opioid taper, fentanyl patch.

\section{BACKGROUND}

Inflammatory Bowel Disease (IBD) is made up of two major diseases: ulcerative colitis (UC) and Crohn's disease (CD). These diseases are characterized by chronic, idiopathic inflammation of the gastrointestinal (GI) tract. A 2007 study analyzing insurance claims in the United States estimated the prevalence of CD and UC in adults to be 201 and 238 per 100,000 individuals, respectively (1). The prevalence of IBD exceeds $0.3 \%$ in North America with an accelerating incidence in newly industrialized countries (2).

IBD is often associated with abdominal pain that can be difficult to control. There are several potential mechanisms for this pain including partial bowel blockage, distension, or significant inflammation. This pain is further complicated by the higher prevalence of psychiatric comorbidities such as anxiety and depression in IBD patients (3). A previous study found that $87.9 \%$ of patients with IBD reported pain. Pain levels were similar between CD and UC patients, and both showed a statistically significant reduction in health-related quality of life when compared to healthy controls (4).

A substantial portion of patients with IBD are prescribed opioids despite limited benefits. Targownik et al (5) examined a large-scale cohort of individuals with IBD who were prescribed opioids in Manitoba. This study concluded that IBD is an independent risk factor for becoming a heavy opioid user, even when controlling for psychiatric comorbidities. Furthermore, it was found that $5 \%$ of individuals with IBD became heavy opioid

From: 'Department of Anesthesiology, Perioperative Care, and Pain Medicine at NYU Langone Health, New York, NY; ${ }^{2}$ Department of Emergency Medicine at NYU Langone Health, New York, NY; ${ }^{3}$ Department of Surgery at NYU Langone Health, New York, NY

Corresponding Author: Samuel Ang, MD, E-mail: samuel.ang@nyulangone.org Disclaimer: There was no external funding in the preparation of this manuscript.

Conflict of interest: Each author certifies that he or she, or a member of his or her immediate family, has no commercial association (i.e., consultancies, stock ownership, equity interest, patent/licensing arrangements, etc.) that might pose a conflict of interest in connection with the submitted manuscript. Accepted: 2021-02-08, Published: 2021-06-14 
users (defined as continuous use of opioids for 30 days at a dose exceeding 50 morphine milligram equivalents per day) within 10 years of diagnosis. Importantly, heavy opioid use was also strongly predictive of mortality (5). Given these findings, it is important for clinicians to exercise caution when starting patients with IBD on opioids.

The evidence for long-term opioid use in chronic non-cancer pain is weak (6). Guidelines regarding the tapering of long-term opioid therapy for chronic pain are still being developed, with no clear consensus about optimal taper schedule (7). Per the American College of Occupational and Environmental Medicine's guidelines, dosage can be decreased by up to $50 \%$ daily when it is determined the opioids are no longer necessary (8). However, many clinicians adhere to slower tapering regimens ( $10 \%$ or less per week) in order to minimize withdrawal symptoms (9). Although opioid withdrawal is associated with nonlethal symptoms, these symptoms, in conjunction with psychiatric comorbidities, can present a significant barrier to successful tapering (7).

To date, there has been no evidence regarding the use of epidural analgesia for rapid opioid taper. We present a case in which a thoracic epidural catheter with patient-controlled epidural analgesia (PCEA) was utilized as part of a multimodal pain management regimen to rapidly taper a patient with chronic abdominal pain who was on a high-dose transdermal fentanyl patch. The patient had an adverse event-free, successful taper with minimal withdrawal symptoms. This manuscript adheres to Health Insurance Portability and Accountability Act privacy guidelines and consent was obtained from the patient for the publication of this case report.

\section{CASE DESCRIPTION}

A 36-year-old man with a history of UC with a stage III J-pouch was admitted to the hospital with recurrent partial bowel obstruction. He had been managed for the past 10-12 years by a pain physician at an outside hospital with a fentanyl transdermal patch with a documented regimen of $75 \mu \mathrm{g} / \mathrm{hr}$. The primary team consulted the pain management team for assistance in tapering the current opioid regimen given concerns that long-term opioid analgesics were contributing to recurrent episodes of bowel obstruction. The patient had attempted outpatient weaning of his fentanyl patch in the past, but only tolerated a reduction of $12 \mu \mathrm{g} / \mathrm{hr}$ monthly without encountering intolerable withdrawal symptoms. His physical exam was significant for a moderately tender abdomen and minimal bowel sounds. Labs were within normal limits.

The pain management team discussed the option of placing an epidural catheter to help control the patient's pain while he was admitted with the long-term goal to eliminate reliance on the fentanyl transdermal patch, improve gastrointestinal motility and discharge on minimal to no opioids. The psychiatry liaison service was consulted and diagnosed the patient with complex opioid dependence and anxiety in the setting of weaning opioid analgesics and recommended the use of gabapentin as an adjunctive therapy for pain and lorazepam for the patient's anxiety.

On hospital day 3, a thoracic epidural at T11-T12 was placed, at which time the fentanyl patch was removed. Subsequently, a fentanyl $(3 \mu \mathrm{g} / \mathrm{mL})$ - ropivacaine $(0.25$ $\mathrm{mg} / \mathrm{mL}$ ) PCEA regimen was started at a basal rate of $15 \mathrm{~mL} / \mathrm{hr}(45 \mu \mathrm{g} / 3.75 \mathrm{mg})$, bolus dose of $4 \mathrm{~mL}(12 \mu \mathrm{g} / 1$ $\mathrm{mg})$, and a lockout period of 10 minutes (15/4/10), with a possible max dose of $39 \mathrm{~mL}(117 \mu \mathrm{g} / 9.75 \mathrm{mg})$ of fentanyl/ropivacaine every hour. Overnight, he started to report feelings of withdrawal symptoms described as "shaking, anxieties." The symptoms were attributed to feelings of anxiety, as per the psychiatry liaison service. The PCEA dosing was significantly higher than his home fentanyl transdermal dosing, lowering suspicion for clinical opioid withdrawal.

On day 8, the patient underwent an anal exam under anesthesia and pouchoscopy (both are minimally painful procedures) with no requirement of up-titration postoperatively. On day 11, the patient's PCEA was gradually down-titrated to $18 \mathrm{~mL} / \mathrm{hr}(54 \mu \mathrm{g} / 4.5 \mathrm{mg}$ max dose/hr) and discussions were started on removing the epidural in the next 1-2 days. On day 12 , he continued to have no clinical signs of withdrawal and it was determined safe to discontinue his epidural.

The patient was ultimately down-titrated to $9 \mathrm{~mL} / \mathrm{hr}$ (27 $\mathrm{\mu g} / 2.25 \mathrm{mg} \max$ dose/hr) and was transitioned to oral opioids in preparation for discharge. The patient's recommended opioid taper regimen was as follows: oxycodone $15 \mathrm{mg}$ by mouth every 6 hours scheduled for 2-3 days, followed by $10 \mathrm{mg}$ every 6 hours scheduled for 2-3 days, followed by $5 \mathrm{mg}$ every 6 hours scheduled for 2-3 days followed by $5 \mathrm{mg}$ every 12 hours scheduled for 3 days, followed by $1 \mathrm{mg}$ daily scheduled for 3 days, then discontinuation. On day 17 , he was discharged home off opioid medications entirely. He remained motivated and optimistic about the action plan.

After discharge, the patient followed up with his pain 
physician at an outside hospital. A month following discharge he was put back on transdermal fentanyl due to concern for inconsistent absorption of oral oxycodone. The initial transdermal fentanyl patch was started at 25 $\mu \mathrm{g} / \mathrm{hr}$ and 2 weeks later decreased to $12 \mu \mathrm{g} / \mathrm{hr}$ resulting in a significantly lower dose than he was using just 2 months prior $(75 \mu \mathrm{g} / \mathrm{hr})$.

\section{DISCUSSION}

The tapering of opioids in patients is very individualized and often requires lengthy regimens. Long-term goals in opioid tapers include improving quality of life, minimizing withdrawal symptoms, and decreasing morbidity and mortality. In 2016, the Centers for Disease Control and Prevention recommended starting tapers at $10 \%$ of the original dose per week until the lowest dose of opioid has been achieved, followed by extending the time interval between doses until the patient is completely weaned off (9). Compared to this standard opioid taper regimen, our patient was tapered much more rapidly.

One proposed means of opioid tapering is through the use of opioid antagonists. In a randomized-controlled trial by O'Connor et al (10), patients underwent heroin detoxification in a primary care setting; a greater percentage of patients in the naltrexone and buprenorphine groups were successfully tapered (10). There have also been case reports linking ketamine infusions to successful, rapid opioid tapers. In one case report, a patient taking 330 daily morphine milligram equivalents was able to be successfully tapered off of opioids with minimal withdrawal symptoms after a 5-day ketamine infusion (11). Although various methods of tapering are being studied, the use of epidural analgesia has not yet been elucidated and may be an effective method. While there have been no data in regards to using epidurals in this manner, there has been evidence suggesting the utility of nerve blocks in opioid tapers. In a case report by Sakamoto et al (12), a patient with pancreatic cancer receiving $240 \mathrm{mg}$ oxycodone daily was able to be rapidly tapered to $80 \mathrm{mg} / \mathrm{d}$ using a celiac plexus block (12). This suggests that nerve blocks, and potentially neuraxial anesthesia, may be utilized for rapid opioid tapering.

Our patient had a history of recurrent bowel obstructions and had been using transdermal fentanyl patches for 10-12 years prior to admission. The surgical team believed that high-dose opioids were contributing to these recurring obstructions. Since sympathectomy from neuraxial anesthesia increases GI motility, epidural analgesia was selected to both improve GI motility while also attempting to rapidly taper opioid usage (13). The epidural regimen started with a possible max dose of $117 \mu \mathrm{g}$ of fentanyl every hour, which was significantly higher than the patient's outpatient regimen $(75 \mu \mathrm{g} /$ $\mathrm{hr}$ at time of admission). The patient was subsequently able to be tapered at an average rate 6-9 $\mu \mathrm{g}$ of fentanyl per day over a 10-day period. Although the patient was eventually placed back on transdermal fentanyl as an outpatient, he was successfully tapered to a significantly lower dose ( $12 \mu \mathrm{g} / \mathrm{hr}$ ) with the temporary use of thoracic epidural analgesia.

The unique use of epidural infusions for rapid opioid tapering has not been studied. Long-term follow-up would be needed to better understand patient comfort as well as sustainability. With the deleterious effects of opioids on quality of life as well as patient morbidity and mortality, various methods of rapid opioid tapering should be further explored. Continued research can ultimately lead to alternative approaches to weaning therapies and individualized regimens for opioid substitution.

\section{CONCLUSION}

For patients with IBD and other similar GI diseases, significant and chronic pain can often lead to the prescription of high-dose opioid regimens. While pain control is important, opioids can subsequently decrease GI motility and contribute to recurrent bowel obstructions in these patients. Epidural analgesia may be utilized in these patient populations to increase gut motility while also providing a means of rapidly tapering opioid consumption. Based on our patient's favorable results, it would be beneficial to further explore and discuss the use of epidurals in the setting of rapid opioid taper.

\section{Author Contributions}

Samuel P. Ang: This author aided in manuscript preparation.

Martha L. Castro: This author aided in manuscript preparation.

Michael Montuori: This author aided in manuscript preparation.

Shengping Zou: This author aided in manuscript preparation.

Feza Remzi: This author aided in manuscript preparation.

Steven Calvino: This author aided in manuscript preparation and conceived the study. 


\section{REFERENCES}

1. Kappelman MD, Rifas-Shiman SL, Kleinman K, et al. The prevalence and geographic distribution of Crohn's disease and ulcerative colitis in the United States. Clin/ Gastroentero Hepatol 2007; 5:1424-1429.

2. Ng SC, Shi HY, Hamidi N, et al. Worldwide incidence and prevalence of inflammatory bowel disease in the 21st century: A systematic review of population-based studies. Lancet 2017; 390:27692778.

3. Bielefeldt K, Davis B, Binion DG. Pain and inflammatory bowel disease. Inflamm Bowel Dis 2009; 15:778-788.

4. Schirbel A, Reichert A, Roll S, et al. Impact of pain on health-related quality of life in patients with inflammatory bowel disease. World J Gastroenterol 2010; 16:3168-3177.

5. Targownik LE, Nugent Z, Singh H, Bugden S, Bernstein CN. The Prevalence and predictors of opioid use in inflammatory bowel disease: A population-based analysis. Am J Gastroenterol 2014; 109:1613-1620.

6. Noble M, Treadwell JR, Tregear SJ, et al. Long-term opioid management for chronic noncancer pain. Cochrane Database Syst Rev 2010; CD006605.

7. Berna C, Kulich RJ, Rathmell JP. Tapering long-term opioid therapy in chronic noncancer pain: Evidence and recommendations for everyday practice. Mayo Clin Proc 2015; 90:828-842.

8. Hegmann KT, Weiss MS, Bowden $\mathrm{K}$, et al. ACOEM practice guidelines: Opioids for treatment of acute, subacute, chronic, and postoperative pain. J Occup Environ Med 2014; 56:e143-159.

9. Dowell D, Haegerich TM, Chou R. CDC guideline for prescribing opioids for chronic pain — United States, 2016. MMWR Recomm Rep 2016; 65.

10. O'Connor PG, Carroll KM, Shi JM, Schottenfeld RS, Kosten TR, Rounsaville BJ. Three methods of opioid detoxification in a primary care setting. A randomized trial. Ann Intern Med 1997; 127:526530.

11. Ocker AC, Shah NB, Schwenk ES, Witkowski TA, Cohen MJ, Viscusi ER. Ketamine and cognitive behavioral therapy for rapid opioid tapering with sustained opioid abstinence: A case report and 1-year follow-up. Pain Pract 2020; 20:95-100.

12. Sakamoto A, Takayama H, Mamiya K, Koizumi T. Opioid withdrawal presenting only nausea during tapering of oxycodone after celiac plexus block: A case report. Ann Palliat Med 2016; 5:67-70.

13. Steinbrook RA. Epidural anesthesia and gastrointestinal motility. Anesth Analg 1998; 86:837-844. 